\title{
Adaptive Carrier Recovery using Multi-Order DPLL for Mobile Communication Applications
}

\author{
Byungjin Chun, Beomsup Kim and Yong Hoon Lee \\ Dept. of Electrical Engineering \\ Korea Advanced Institute of Science and Technology \\ 373-1 Kusong-dong, Yusong-gu, Taejon, 305-701, Korea \\ TEL : 82-42-869-3437 FAX : 82-42-869-3410
}

\begin{abstract}
A salient adaptive carrier recovery with a multiorder Digital Phase-Locked Loop (DPLL) based on Kalman filtering is presented in this paper. Using optimal loop gain sequence, that is Kalman gain sequence, for various optimization criteria, the proposed DPLL achieves fast phase acquisition and low phase jitter. Especially, the loop gain sequence optimized under the Least Squares (LS) criterion shows enough phase tracking capability under severe fading and can be used for mobile communication applications. Finally, the multi-order DPLL allows robust stability and bandwidth control and achieves zero phase offset.
\end{abstract}

\section{INTRODUCTION}

In many codeless communication applications, Phase-Locked Loop (PLL) has been used for carrier recovery in a receiver. Among the applications, the digital mobile communication requires sophisticated PLL design for carrier recovery because of high dynamic source condition and fast fading channel environment. In general, two characteristics of the PLL are required when no fading exists; fast phase acquisition to shorten training period in initial locking mode and small phase jitter variance to reduce the bit error rate in steady state. However, these characteristics are contradictory since fast phase acquisition requires wide loop bandwidth and small phase jitter requires narrow loop bandwidth [1]. In Digital Phase-Locked Loop (DPLL), it is possible to control loop gain adaptively. So, the above characteristics can be achieved together by adjusting loop bandwidth (or, loop gain) adaptively in an effort to minimize the phase jitter variance at any given time instance. Such aftempts have been made up to the second-order DPLL [2] [4]. Under the fading environment, the DPLL is also required to track the phase/frequency fluctuation. To achieve this, Least Squares (LS) or Recursive Least Squares (RLS) adaptive algorithm has been adopted for the second-order DPLL for moderate fading condition [3]. But, for more severe dynamic situation with phase acceleration or more, higher-order DPLL is necessary to track the dynamics.

This paper generalizes the second-order DPLL idea up to any higher-order DPLL in order to meet the above situation using Kalman filtering concept [5]. The approach is based on the observation that DPLL and Kalman filter have similar structures and the optimum loop gain sequence minimizing phase noise variance at each time instance can be obtained by finding Kalman gain sequence with a properly chosen system and observation model.

Two different criteria to get the optimal loop gain sequences are used for different channel environments. For stationary or slowly varing channel, MMSE (Minimum Mean Square Error) criterion is used and for non-stationary or fading channel, LS (Least Squares) criterion is used. To reduce hardware and computational requirements, its recursive version, RLS (Recursive Least Squares) criterion can be used instead.

In section 2, the multi-order DPLL Kalman filter model is explained and its characteristics are described. In section 3, optimal loop gain sequences are derived under the MMSE and LS criteria. In section 4 , computer simulations verify that the proposed DPLL shows satisfactory acquisition and tracking performances under fast fading channel environment. Finally, conclusions are given in section 5 .

\section{Set Up of Kalman Filter EQUATIONS}

As a typical higher-order system, the third-order carrier generation system with phase offset $\theta_{0}$, frequency offset $\omega_{0}$ and phase acceleration offset $\eta_{0}$ is considered at the transmitter. To apply the Kalman filtering method to the DPLL, we need the system model of the transmitted carrier and the observation model at the receiver. Although we can form the system model using the state variables as phase $\theta(k)$, 


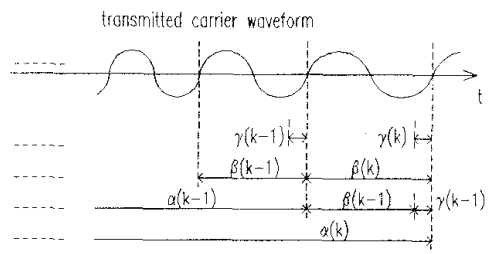

Figure 1: Definitions of transmitted carrier states (Constant deceleration is assumed in this figure.).

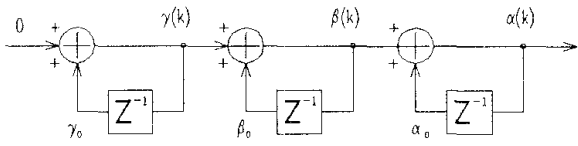

Figure 2: System model for transmitted carrier.

frequency $\omega(k)$ and phase acceleration $\eta(k)$ respectively at $\mathrm{k}$-th sampling point, we prefer using zerocrossing time $\alpha(k)$, period $\beta(k)$ and period increment $\gamma(k)$ of $\mathrm{k}$-th sinusoid as state variables since it makes the problem simple (Figure 1). Also, we can easily relate $\theta_{0}, \omega_{0}, \eta_{0}$ to zero-crossing time offset $\alpha_{0}$, period offset $\beta_{0}$, period increment offset $\gamma_{0}$ respectively. In this manner, the carrier recovery problem falls into an estimation of $\alpha_{0}, \beta_{0}$ and $\gamma_{0}$ from noisy observation at the receiver.

By defining state vector $X(k) \equiv[\alpha(k) \beta(k) \gamma(k)]^{T}$, the state transition equation with state transition matrix $A$ can be expressed as follows. (Also, refer to Figure 2.)

$$
X(k)=A X(k-1)
$$

where

$$
A=\left[\begin{array}{lll}
1 & 1 & 1 \\
0 & 1 & 1 \\
0 & 0 & 1
\end{array}\right]
$$

The transmitter carrier generated according to the system model suffers additive distortion due to channel noise and multiplicative distortion due to fading effect in channel. So the zero-crossing time of received carrier will show a considerable amount of jitter. When the channel is stationary with Additive White Gaussian Noise (AWGN) only, the estimation of source states can be solved statistically. However, when the channel is non-stationary with fading, we are forced to rely on adaptive algorithm. To show the idea of our approach which is directly related to Kalman filtering, we assume that channel distortion is due to only stationary AWGN first, then extend it to non-stationary case later.

The receiver should also be the third-order system in order to track source states from incoming carrier. After measuring zero-crossing time $\alpha_{i}(k)$ of the received carrier, we get its period $\beta_{i}(k)$ by differencing $\alpha_{i}(k)$ and get its period increment $\gamma_{i}(k)$ by again differencing $\beta_{i}(k)$ (Figure 3 ). If we define observation vector $Y(k) \equiv\left[\alpha_{i}(k) \beta_{i}(k) \gamma_{i}(k)\right]^{T}$, the observation equation with observation matrix $C$ can be expressed as (3).

$$
Y(k)=C X(k)+V(k)
$$

where

$$
\begin{aligned}
C & =\left[\begin{array}{lll}
1 & 0 & 0 \\
0 & 1 & 0 \\
0 & 0 & 1
\end{array}\right] \\
V(k) & \equiv\left[\begin{array}{c}
v(k) \\
v(k)-v(k-1) \\
v(k)-2 v(k-1)+v(k-2)
\end{array}\right]
\end{aligned}
$$

Here, $v(k)$ is AWGN with variance $\sigma_{v}^{2}$ and $V(k)$ is $o b$ servation noise vector with observation noise covariance matrix $R \equiv E\left[V(k) V(k)^{T}\right]$, which is calculated as $(6)$.

$$
R=\sigma_{v}^{2}\left[\begin{array}{lll}
1 & 1 & 1 \\
1 & 2 & 3 \\
1 & 3 & 6
\end{array}\right]
$$

Given the state transition equation (1) and the observation equation (3), we can estimate source states with minimum error variance at any instance $k$ using the following Kalman filter recursion equations (7), (8).

$$
\begin{array}{r}
\hat{X}(k)=\hat{X}(k \mid k-1)+K(k)(Y(k)-C \hat{X}(k \mid k-1)) \\
\hat{X}(k+1 \mid k)=A \hat{X}(k)
\end{array}
$$

where

$$
\begin{aligned}
& \hat{X}(k) \equiv\left[\begin{array}{lll}
\hat{\alpha}(k) & \hat{\beta}(k) & \ddot{\gamma}(k)
\end{array}\right]^{T} \\
& \hat{X}(k+1 \mid k) \equiv[\hat{\alpha}(k+1 \mid k) \hat{\beta}(k+1 \mid k) \hat{\gamma}(k+1 \mid k)]^{T}(10) \\
& K(k) \equiv\left[\begin{array}{lll}
K_{11} & K_{12} & K_{13} \\
K_{21} & K_{22} & K_{23} \\
K_{31} & K_{32} & K_{33}
\end{array}\right]
\end{aligned}
$$

Here, $\hat{X}(k), \hat{X}(k+1 \mid k), K(k)$ are filtered state vector, a priori predicted state vector and Kalman gain matrix (or, loop gain matrix) respectively. We can consider stability by combining (7) and (8) to get (12) below.

$\hat{X}(k+1 \mid k)=[A(I-K(k) C)] \hat{X}(k \mid k-1)+A K(k) Y(k) \quad(12)$ where $I$ means Identity matrix. If $K(k)$ has only diagonal parts, the roots of $\operatorname{det}[A(I-K(k) C)]=0$ are all inside unit circle as long as $0<K_{i i}<1(i=$ $1,2,3)$, which means stable system.

Therefore, we choose to use $K(k)$ with diagonal parts only. Figure 3 shows the proposed DPLL implementation according to $(7)$ and (8). It consists of three first-order loops, and each loop estimates corresponding; source state. 


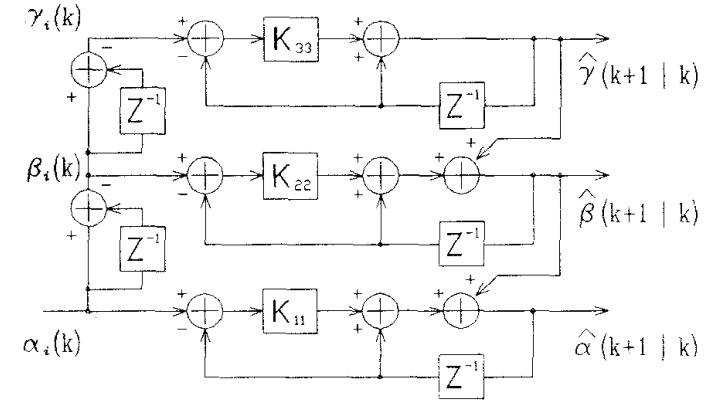

Figure 3: The proposed multi-order DPLL (the thirdorder case).

\section{Optimal Loop Gain SEQUENCES}

For stationary or slowly varying channel environment, MMSE (Minimum Mean Square Error) can be a criterion to find optimal loop gain sequence. We define a priori estimation error covariance matrix $P^{M M S E}(k+1 \mid k)$ as a cost function in MMSE criterion as (13).

$$
P^{M M S E}(k+1 \mid k)=E\left[\mathcal{E}(k+1 \mid k) \mathcal{E}(k+1 \mid k)^{T}\right]
$$

where $\mathcal{E}(k+1 \mid k)$ means a priori estimation error $X(k+1)-\hat{X}(k+1 \mid k)$. Then, minimizing the $(1,1)$ th element of $P^{M M S E}(k+1 \mid k)$, which is a priori zerocrossing time estimation error variance, with respect to diagonal parts of loop gain $K(k)$, we get the $k$-th optimal loop gain in MMSE criterion $K^{M M S E}(k)$ as follows.

where

$$
K^{M M S E}(k)=\tilde{M}^{-1} \tilde{L}
$$

$$
\begin{array}{r}
\tilde{M}_{i j}=A_{1 j}\left[C P^{M M S E}(k \mid k-1) C^{T}+U(k \mid k-1) C^{T}\right. \\
\left.+C U(k \mid k-1)^{T}+R\right]_{i j} \\
\tilde{L}_{i 1}=\left[A\left(C P^{M M S E}(k \mid k-1)+U(k \mid k-1)\right)\right]_{1 ;} \\
,(i, j=1,2,3)
\end{array}
$$

Introduction of $U(k \mid k-1) \equiv E\left[V(k) \mathcal{E}(k \mid k-1)^{T}\right]$ in $(15),(16)$, is due to the fact that $R(k, l) \equiv$ $E\left[V(k) V(l)^{T}\right]$ is not always zero even for $k \neq l$. This shows a little deviation from the standard Kalman filter equation set. If we know all the statistics of the desired signal and observation, we can get the optimal loop gain sequence by solving (14) with a given initial condition. However, in practice, the statistics are not known at the receiver. Therefore, the suboptimal loop gain sequence is used instead by setting initial value $K^{M M S E}(1)=I$. Here, $K^{M M S E}(1)=I$ means infinite loop bandwidth [2]. The optimal or sub-optimal loop gain sequence $K^{M M S E}(k)$ thus guarantees the fastest phase acquisition in initial locking mode and the lowest phase jitter variance in steady state.

We can extend the above idea to non-stationary channel environment with the same receiver structure by choosing LS (Least Squares) criterion for the optimal loop gain sequence. Now, the nature of problem becomes a adaptive curve fitting to observations. Cost function in LS criterion $P^{L S}(k+1 \mid k)$ is defined as

$$
P^{L S}(k+1 \mid k) \equiv \sum_{l=k+1-L}^{k+1}(Y(l)-\tilde{X}(l))(Y(l)-\tilde{X}(l))^{T}
$$

where observation vector $Y(l)$ corresponds to the desired signal in LS algorithm which is linearly estimated in backward manner with respect to a priori estimation $\hat{X}(k+1 \mid k)$ as $(18)$.

$\tilde{X}(l) \equiv A^{l-k-1} \hat{X}(k+1 \mid k) \quad, \quad(l=k+1-L, \cdots, k+1)(18)$

Here, $L$ means window length. In almost-stationary case, a large number is chosen for $L$. On the other hand, a small number is chosen in rapid phase fluctuation case. Like the MMSE case, the $(1,1)$ th element of $P^{L S}(k+1 \mid k)$ is minimized with respect to the diagonal parts of $K(k)$ to get optimally adapted $k$-th loop gain $K^{L S}(k)$ as shown in (19).

$$
K^{L S}(k)=\bar{M}^{-1} \tilde{L}
$$

where

$$
\begin{aligned}
\tilde{M}_{i 3} & =\sum_{l=k+1-L}^{k+1} A_{1 i}^{l-k} A_{1 j}^{l-k}\left(L_{2}(k) L_{2}(k)^{T}\right)_{1 j} \\
\tilde{L}_{i 1} & =\sum_{l=k+1-L}^{k+1} A_{1 i}^{l-k}\left(L_{1}(l, k) L_{2}(k)^{T}\right)_{1 i} \\
L_{1}(l, k) & \equiv Y(l)-A^{l-k} \hat{X}(k \mid k-1) \\
L_{2}(k) & \equiv Y(k)-\hat{X}(k \mid k-1),(i, j=1,2,3)
\end{aligned}
$$

Using $A^{l-k}$ as (24), $\tilde{M}$ and $\tilde{L}$ can be evaluated easily.

$$
A^{l-k}=\left[\begin{array}{ccc}
1 & (l-k) & \frac{(l-k)(l-k+1)}{2} \\
0 & 1 & (l-k) \\
0 & 0 & 1
\end{array}\right]
$$

Since LS algorithm requires lots of computation and storage, it is difficult for a DSP hardware to do real time processing as the window length $L$ gets large. To relieve it, the RLS (Recursive Least Squares) algorithm may be used instead. RLS algorithm can be obtained in the same manner as LS algorithm if the summation operation $\sum_{l=k+1-L}^{k+1}$ is substituted with $\sum_{l=1}^{k+1} \lambda^{k+1-l}$ making recursive calculation possible. Here, $\lambda$ is the exponential weighting factor which represents memory size $(0<\lambda<1)$. In almoststationary case, $\lambda$ is chosen close to 1 , whereas in rapid phase fluctuation case, $\lambda$ is chosen close to 0 .

The third-order algorithm derived so far can be easily extended to any higher-order case using the same Kalman filter equation set (1), (3), (7), (8), and readily calculable matrices $A, C, R$ in (2), (4), (6). 


\section{Simulation Results}

As an application example, we show BPSK mobile communication system which consists of BPSK modulator, fading channel and corresponding demodulator using the proposed DPLL with the RLS algorithm as shown in Figure 4. Transmitter carrier may have an arbitrary phase and frequency offset and a phase acceleration component. To simulate mobile communication channel, Raileigh fading channel with maximum Doppler frequency $100 \mathrm{~Hz}$ and moderate SNR are assumed. Figure 5 shows rapid tracking onto the phase fluctuation due to fading and considerable phase jitter reduction enough to be used for carrier recovery. Figure 6 shows the tracking performance comparison between the proposed third-order and the second-order DPLL for maximun Doppler frequency $500 \mathrm{~Hz}$. We can verify that the third-order DPLL tracks the rapid phase fluctuation more closely than the second-order DPLL does.

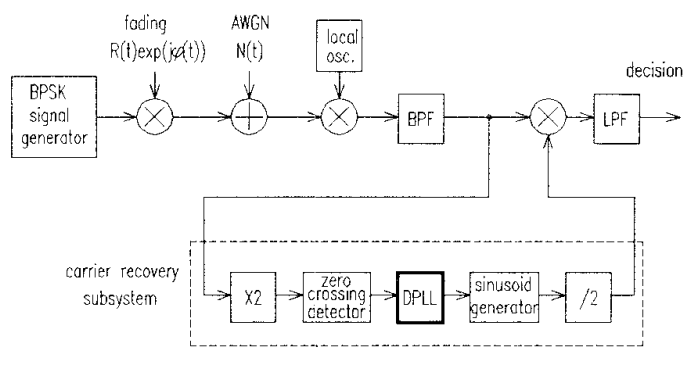

Figure 4: A typical mobile communication system and carrier recovery subsystem (BPSK case).
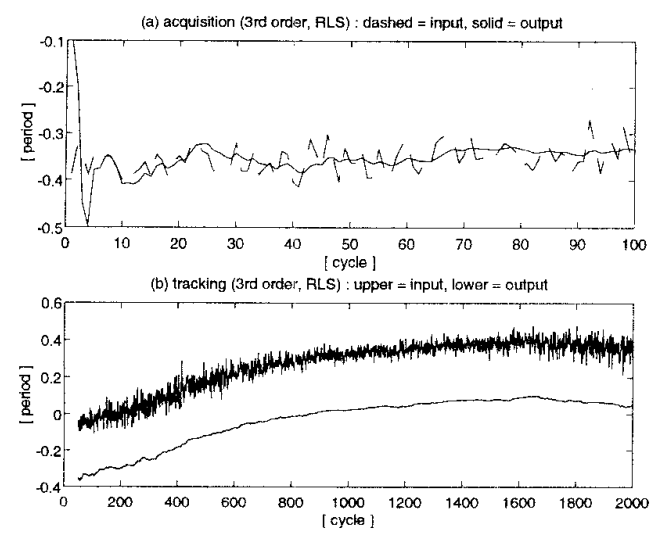

Figure 5: Acquisition and tracking performances of the proposed third-order DPLL under fading channel environment.

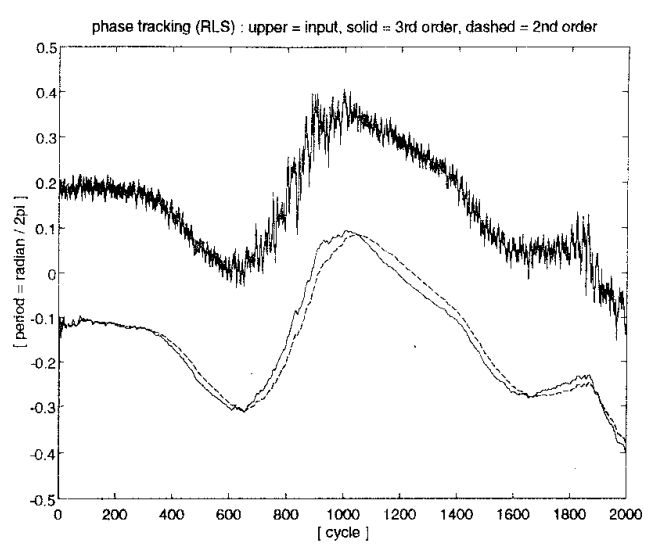

Figure 6: The tracking performance comparison between the third-order DPLL and the second-order DPLL.

\section{CONCLUSION}

A multi-order DPLL with adaptive loop gain control capability is proposed and its optimal loop gain sequence to track rapid phase fluctuation is solved. The DPLL can be suitably used in the carrier recovery subsystem for digital mobile communication applications, especially in severe fading condition.

\section{References}

[1] F. Gardner, Phaselock Techniques, WileyInterscience Publication, 1979.

[2] Beomsup Kim, "Optimal MMSE Gear-shifting Algorithm for the Fast Synchronization of DPLL," in ISCAS, pp. 172-175, 1993.

[3] Beomsup Kim, "Digital Carrier Recovery with Adaptive Dual Loop DPLL for Mobile Communication Applications," in ICASSP, 1993.

[4] P. F. Driessen, "DPLL Bit Synchronizer with Rapid Acquisition Using Adaptive Kalman Filtering Techniques," IEEE Transactions on Communications, vol.42(9), pp.2673-2675, Sep. 1994.

[5] R. G. Brown, Introduction to Random Signal Analysis and Kalman Filtering, Englewood Cliffs, NJ: John Wiley \& Sons, 1983. 